\title{
The Electroforming of Gold
}

\section{A MANUFACTURING TECHNIQUE FOR INTRICATE COMPONENTS}

\section{A. Mohan}

Veco Research BV, Eerbeek, The Netherlands

In the majority of its applications in industry gold is used as a surface layer or coating, but the production of small solid gold components by electroforming is finding a number of applications where intricate parts are required, made with great accuracy in either small or large quantities.

Electroforming may be defined as the technique of producing or reproducing articles by the use of electroplating processes. An electroformed article is the electrodeposit separated from the master or matrix on which it is deposited. It could therefore be said that any metal that can be electrodeposited could in theory be used for electroforming; but as the electroformed article is an object in its own rightthat is independent of the matrix on which it is plated-the physical properties of the electrodeposited metal should be such that when it is removed or separated from its matrix or mandrel it has the strength and coherence to support itself and to perform the task for which it is designed.

In practical terms the electrodeposited metal should be capable of being deposited from controllable electrolytes to give acceptable physical and chemical properties. For this reason the most commonly used metals in electroforming are nickel and copper, but other metals such as silver, platinum, gold, tin, iron and lead have found limited uses.

The process consists basically, of course, of electrolytically depositing the chosen metal on a master or mandrel to the required thickness and then removing the electrodeposited metal from the matrix. Therefore the basic technique can have many possible variations. For the purposes of this article we will divide it into two main types:

(1) Electroforming on a matrix produced or generated by a photographic system-Photoforming.

(2) Electroforming on a mandrel produced by other engineering methods.

The photoforming process is generally used for the production of perforated products like sieves, filters, electron microscope grids, optical apertures, electronic devices, in fact for any comparatively thin electroformed product $(10 \mu \mathrm{m}$ to $200 \mu \mathrm{m})$ where the electrodeposit is made on some areas and not on others in a pattern or design that can be generated or created with great accuracy by photographic means.

This system uses a base plate of a suitable metal which is coated with a photosensitive lacquer. This lacquer, known as a photoresist, can be either a positive working type, meaning that on exposure to actinic light the exposed parts of the lacquer become soluble in a developer, leaving the unexposed areas electrically and chemically inert, or a negative working type where the areas exposed to ultra-violet light are the areas that do not dissolve in the developer. The basic final result, however, is that after the coated plate is exposed to actinic light in close contact with a photomaster and then developed some areas of the metal plate are covered with an insulating layer of lacquer and in other areas bare metal is exposed. If now, after suitably hardening the lacquer to make it fully resistant to the chemical attack of the electrolyte, such a plate is made a cathode in an electroplating bath, metal is deposited on the exposed metal areas of the base plate, thus reproducing by electroforming the exposed pattern on the plate. This electrodeposited metal can, once it has been built up to sufficient thickness, be removed to give the electroformed article.

Simple as this may sound, several factors must be taken into account in this type of electroforming to produce the accurate end product. These are mainly the changes in the shape and geometry of the final product as compared to the photograph from which it is produced due to factors such as the growth characteristics of the deposit, the stress in the deposit, the thermal expansion and the geometry. The basic system has been developed scientifically and commercially by Veco Zeefplatenfabriek among others, and the various factors to be taken into consideration are well understood.

Electroforming on mandrels consists of the deposition of metal on to any mandrel produced by 
From the original drawing of a part to be electroformed on a matrix generated by the photographic system this $X=Y$ co-ordinatograph is used to produce the art work for reduction to the master negative. Tolerances of one micron can be achieved with the stepand-repeat machines employed in the later stages

engineering or other means. This system is used for producing tools, moulds and dies, wave guides, heat exchangers, and certain consumer goods, the list being now almost endless. The mandrels can be either conductive-made from materials like stainless steel, brass, copper, nickel, aluminium or fusible alloys-or nonconductive-made from epoxy resins, waxes, plastics, wood or plaster of paris produced either by casting, machining or carving.

Conductive mandrels generally need a parting layer before electroforming so that the electroform can be easily separated from the mandrel. Alternatively the mandrel can be removed by preferential chemical dissolution.

Non-conductive mandrels are given a conducting surface either by coating them with graphite powder, copper or bronze powder, silver lacquer, or a chemical deposit of copper, nickel or silver. Here again the mandrel is made the cathode in an electroplating bath and the required thickness of metal deposited, the mandrel then being removed from the electrodeposit. This type of electroforming has also been commercially developed to a very advanced stage and a considerable amount of literature is available on the problems, the control factors, the choice of solutions and the techniques of the process, once again mainly for nickel and to a lesser extent for copper and other metals.

The main points to consider in selecting an electrolyte suitable for electroforming are: good throwing power, freedom from nodule formation, a controllable degree of stress in the deposit, stability

Perforated parts such as these gold grids or other components in which the electrodeposit is confined to certain areas only are usually produced on a matrix generated by photographic means

of the solution, and general economic and operational considerations.

Electroforming shows marked advantages in the following areas:

(1) The surface finish of the mandrel is faithfully reproduced, whether it be textured, grained, machined or highly polished.

(2) The dimensions of a precision mandrel can be accurately reproduced.

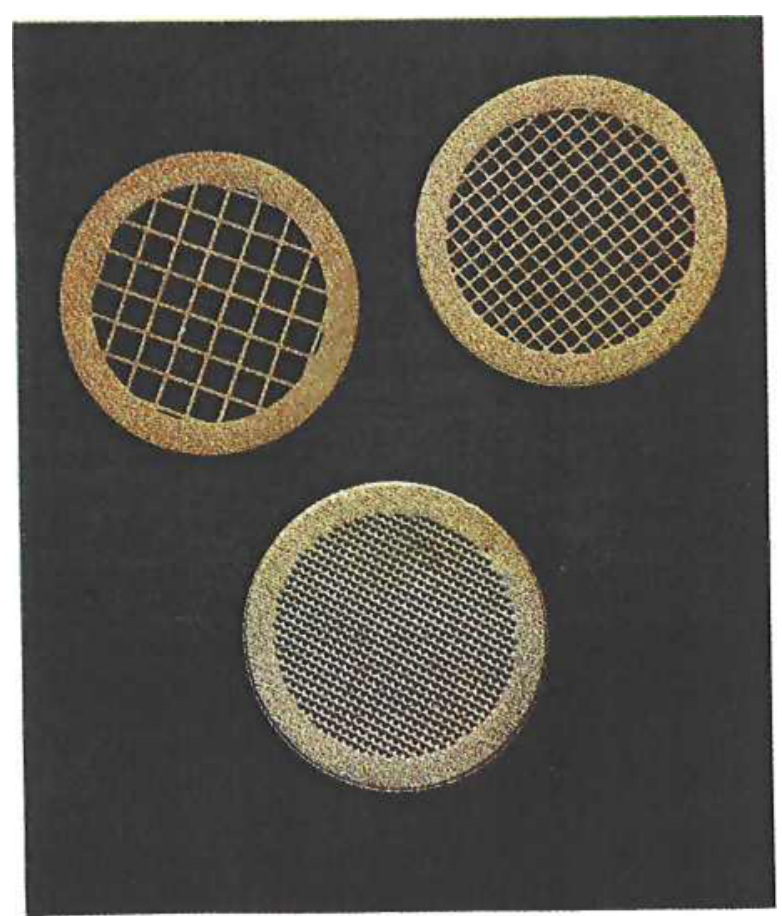


(3) An intricate shape can be produced in a single step-even shapes where a mandrel may be made from several pieces.

(4) More than one identical electroform can often be made from one mandrel.

(5) Highly accurate and complicated products can be made by the photoforming techniques.

In the industrial and commercial development of the electroforming process the choice of metal has generally been governed by the cost of the metal and the electrolyte, the properties of the metal obtainable, the control of the properties of the deposit, the availability of suitable electrolytes and their ease of control, and last but not by any means the least the state of knowledge about these factors and their application to electroforming.

At present the most commonly used metals in the electroforming industry are nickel and copper, with which most of the development in the electroforming industry has been done. Here the control and development of electrolytes and the knowledge of the physical and chemical properties of the deposit are better understood and publicised. Metals such as gold, silver and platinum are of course widely used in the plating industry because of their remarkable and unique properties, and it is the properties that recommend them for certain electroforming uses.

\section{Applications of Gold Electroforms}

Gold electroforming is at present carried out to a limited extent and a number of examples can be given.

Almost certainly the best known example of electroforming in gold was the crown with which the Prince of Wales was invested at Carnarvon in 1969. Here electroforming was chosen as the technique for the reproduction of the unusual surface texture of the original design. The crown was electroformed on an epoxy resin master produced from the original wax model. The electroforming operation required two and a half days, the thickness of the electroform ranging from 0.030 to 0.040 inch.

The electroforming of models of the moon in gold to commemorate the first landing on the moon has been described by D. R. Mason (1). In this case the electroforming was done in two halves on a p.v.c. master, the two sections being subsequently joined by soldering.

Other uses of gold electroforms have been described in the literature. Slip rings have been produced to a thickness of 0.015 inch by depositing gold on to grooves in a plastic cylinder having the lead wires embedded into it. Electrodes for cathode ray tubes, generally made from nickel, have been produced for special purposes in gold from a cyanide type solution by depositing on to a grooved copper plate of the required shape. The copper matrix was then dissolved away, leaving the gold electroformed mesh.

In dental work, again, gold electroforming has been used to form the model of a tooth for the fitting of a crown or inlay.

At Veco electron microscope grids in gold are produced in great quantities by the photoforming technique. A nickel or stainless steel plate is coated with a photoresist and the desired pattern of the grid exposed on it. After developing and post baking, gold is deposited from a low stress gold solution to the required thickness and the grids are then removed from the matrix.

Another similar range of products at Veco are the aperture diaphragms for electron microscopy. These consist of a thin gold film ( 25 to $30 \mu \mathrm{m}$ ) mounted on a ring. The gold film has in its centre a well defined and accurate hole of $5,10,15$ or $25 \mu \mathrm{m}$ diameter. These can be made by producing the gold discs of the required thickness on a photomatrix on a brass or phosphor bronze plate. A low-stress acid gold solution is used and the brass or phosphor bronze is then etched away in a ferric chloride solution, using the gold as an etch resist so that only a supporting ring is left.

A low stress acid solution containing gold chloride and hydrochloric acid or a proprietary bright gold can be used if the proper resist is chosen and if the thickness is not very great (10 to $25 \mu \mathrm{m})$. In certain applications a gold alloy containing a small percentage of nickel or cobalt can also be used.

The fact that in spite of the very attractive properties of gold the electroforming of gold and its alloys is as yet very little practised commercially can and may be due to two reasons. First, of course, gold and its alloys are costly and electroforming requires larger thicknesses by comparison with plating. Secondly, there is in general a lack of information on the production of thick gold deposits and of solutions suitable for their production.

\section{Choice of Electrolyte}

Stress in deposits must be carefully controlled and low stress deposits in comparatively high thicknesses are necessary for electroforming. Ferrocyanidechloride type solutions have been suggested by a number of workers. Paweck and Weiner (2) suggest that thick deposits may be obtained from a solution obtained by boiling potassium ferrocyanide solution with gold cyanide; Kutnetsova (3) proposes a solution of gold as chloride, potassium ferrocyanide and potassium carbonate operated at $30^{\circ}$ to $50^{\circ} \mathrm{C}$ with a current density of $0.1 \mathrm{~A} / \mathrm{dm}^{2}$, while Yampolsky (4) uses a solution of chlorauric acid and potassium ferrocyanide. Korbelak (5) developed a ferrocyanide 


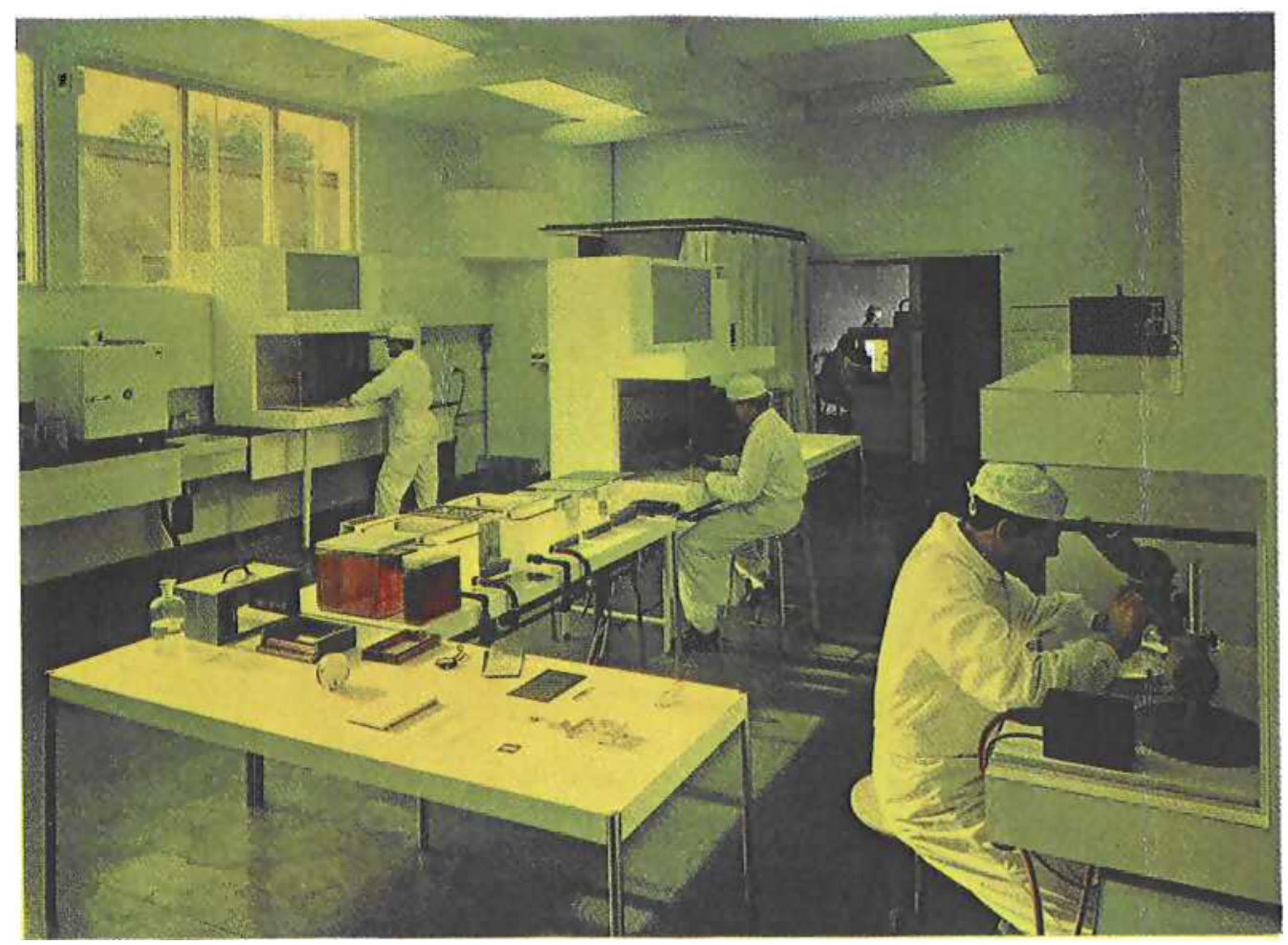

While the production of electroforms in nickel and copper is carried out in a large scale production plant at Veco, precision electroformed components in gold and other less common metals are produced in this dust-free and air-conditioned operating area

solution containing potassium gold cyanide and potassium cyanide which is claimed to be used in American practice at $85^{\circ} \mathrm{C}$ and a current density of $3.5 \mathrm{~A} / \mathrm{dm}^{2}$.

Gold cyanide electrolytes have been used extensively for the production of thick deposits, although they can give rise to problems in the photoforming process because of their tendency to attack the resists. Seegmiller and Gore (6) suggest the use of turkey red oil to modify a cyanide solution and have produced coating 20 to $30 \mathrm{mil}$ ( 500 to $750 \mu \mathrm{m}$ ) thick for the protection of nuclear reactors. Neutral cyanide solutions produced by supply houses have been used in gold electroforming of $25 \mu \mathrm{m}$ and over, but no specific data are available for electroforming purposes.

In the field of thick gold deposits several other types of electrolytes such as potassium gold cyanide with ethylene guanidine and formic acid proposed by Yamamara (7) are reported to give bright, crack free deposits of up to $15 \mu \mathrm{m}$.

In principle the sulphite gold solutions are ideal for electroforming, and have been commercially cmployed. A typical formulation would be:

Sodium gold sulphite

Potassium phosphate

Sodium sulphite

Arsenious oxide

$10 \mathrm{~g} / \mathrm{l}$

$3 \circ \mathrm{g} / 1$

$50 \mathrm{~g} / \mathrm{l}$ $30 \mathrm{mg} / \mathrm{l}$

This has a pH of 9 to 10 and is operated at $50^{\circ} \mathrm{C}$ and a current density of 0.1 to $0.6 \mathrm{~A} / \mathrm{dm}^{2}$.
The sulphite solutions give pure, relatively hard and fully bright deposits with good levelling properties, but there is a tendency to build up free sulphite and sulphate and the life of the solution may be limited and it may indeed be harmful for electroforming purposes.

It is perhaps fair to conclude from this survey that the use of gold in the electroforming industry is small when compared with that of nickel and copper -it would be unreasonable to expect otherwise-but the fact that gold and gold alloys are not as widely used as they perhaps ought to be in view of some of their remarkable properties may in large part be due to the lack of knowledge and information about gold electrolytes suitable for electroforming. Once this information is made available, the electroforming industry will no doubt be better able to exploit the advantages that gold and gold alloys so patently offer.

\section{References}

1 D. R. Mason, Electroplating Metal Finishing, 1969, 22, 8

2 R. Paweck and R. Weiner, Z. Elektrochem., 1930, 36, 972

3 A. N. Kutnetsova, Med. Prom. S.S.R., 1958, 12, 46

4 A. M. Yampolsky, Electroplating Metal Finishing, 1963, 16, (3), 76

5 A. Korbelak, "Metal Finishing Guidebook and Directory", 1969

6 R. Seegmiller and J. K. Gore, Proc. Am. Electroplaters' Soc., 1960, 47, 74

7 K. Yamamara, U.S. Patent, 3,475,290, (1969) 